\title{
Beiträge zur Sedimentologie der Weser- und Elbästuare
}

\author{
HANS LÜNEBURG \\ Institut für Meeresforschung, Bremerbaven
}

\begin{abstract}
Contributions to the sedimentology of Weser and Elbe estuaries. In the preestuaries of the rivers Weser and Elbe (i. e. in the area west of "Elbe 1" and southeast of "P 12" as well as in the northwestern part of the "Nordergründe" and in the "Alte Weser"), the effects of estuary river water on the underlying sediment surfaces have been studied. Another aspect investigated was the effect of eroded Pleistocene horizons on the iron chemistry of these sediments. These horizons are located in the area of the pre-estuaries of both rivers at a depth of more than $20 \mathrm{~m}$ below SKN. The oolitic iron-containing sand grains were quantitatively measured using a special counting method. They are spread out according to the hydrodynamics and bottom dynamics in the pre-estuary of the "Alte Weser" and in additional troughs formed by erosion in the Pleistocene of the estuaries. Thus they represent convenient and reliable indicators for the sediment migration in these troughs; moreover, they suggest the existence of such troughs, which are found only in the southeastern part of the North Sea. In general, it was possible to establish that the sediment areas located beneath the typical bodies of estuary water are especially eutrophic, containing $50 \mathrm{mg}$ and more of $\mathrm{PO}_{4}$ phosphorus per $\mathrm{kg}$ dry sediment, 10 to $20 \%$ total organic substance and 10 to $20 \%$ colloidal iron. These data represent values equivalent to the third power of the respective concentrations in the sea water. - The variations in the chemistry of the interlaying sand zones in the "Nordergründe" concern primarily the iron factor and are mainly affected by the Pleistocene horizons.
\end{abstract}

\section{EINLEITUNG}

Im Anschluß an frühere systematische, hydrographische Untersuchungen über die Wassermischvorgänge im Seegebiet vor den Weser- und Elbmündungen (vorgetragen auf dem Dritten Meeresbiologischen Symposion im Oktober 1962) wurde festgestellt, daß sich die großräumige Verteilung der Weser- und Elbwasserkörper in dem fraglichen Gebiet in den Eigenschaften der oberflächennahen Sedimente widerspiegelt. Das heißt, daß man z. B. unter der aus der Alten Weser (nördlicher Arm der Wesermündung) bis in das Gebiet des Feuerschiffes P 12 setzenden Weserwasserzunge und unter der aus der Außenelbe in Richtung Helgoland abströmenden Elbwasserzunge entsprechende Zonen feinkörnigeren Sedimentes um $50 \mu$ und weniger findet, deren chemische Eigenschaften auf die Beeinflussung aus der Elbmündung und, z. T. mit besonderer Prägung, aus der Alten Weser hinweisen. 


\section{ERGEBNISSE}

Ehe der besondere Einfluß aus der Alten Weser mit seinen natürlichen Gegebenheiten im einzelnen erläutert wird, soll der generelle Weser-Elbeinfluß auf die Sedimente nordwestlich der Nordergründe an Hand einer Darstellung des Gehaltes an $\mathrm{PO}_{4}-\mathrm{P}$ in $\mathrm{mg} / \mathrm{kg}$ Trockensediment erörtert werden (Abb. 1). - Bekanntlich stellt der Phosphat-Phosphor einen repräsentativen Indikator nicht nur für die Eutrophierung des Seewassers vor den Astuaren, sondern auch der dortigen oberflächennahen Sedimentschichten dar.

Zur Bestimmungsmethode des Phosphat-Phosphors in Brack- und Marinsedimenten sei kurz folgendes erwähnt: $\mathrm{Da}$ das vor der Sedimentierung der Sinkstoffe existente kolloide Eisen im Brackwassergebiet in wesentlich höheren Konzentrationen

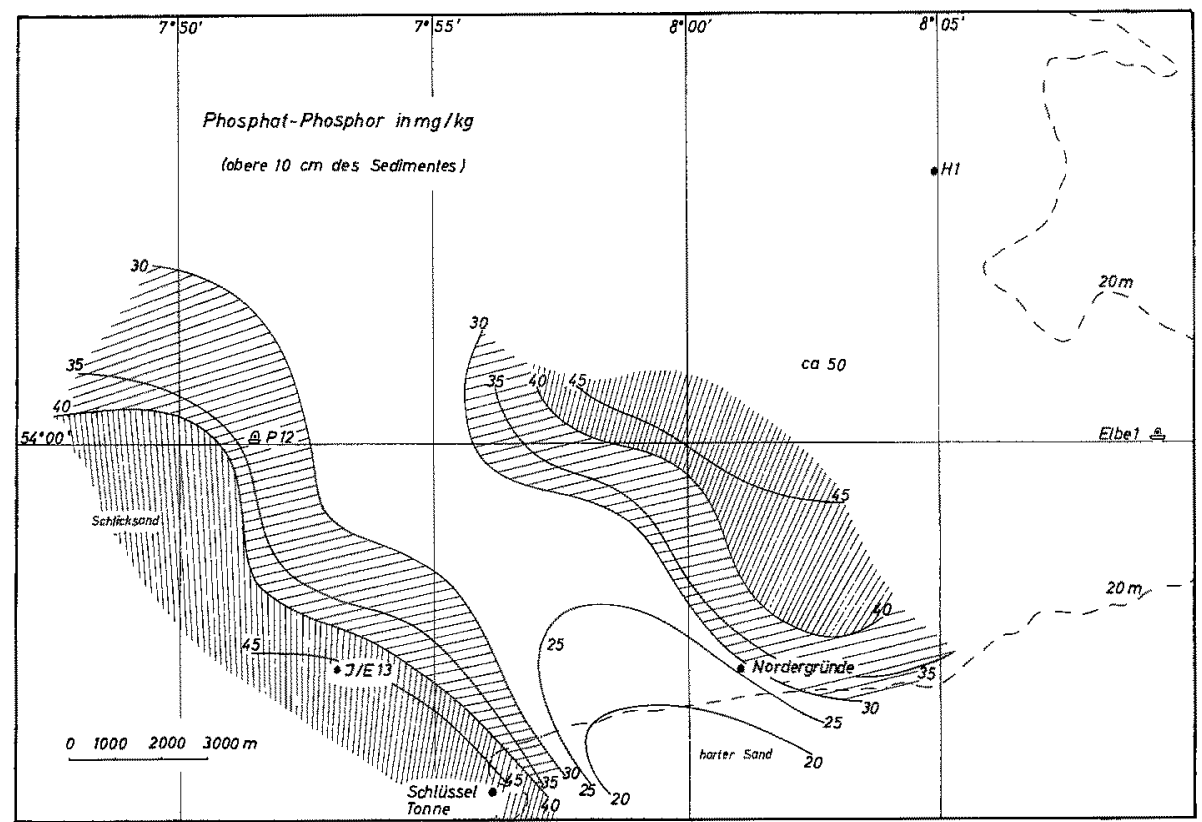

Abb. 1: $\mathrm{PO}_{4}$-P-Gehalt an der Sedimentoberfläche im Vorästuar von Elbe und Weser

als der Phosphat-Phosphor vorkommt (etwa einer Zehnerpotenz), und da ferner das Eisenphosphat im Wasser besonders schwer löslich ist, wird der anorganische Phosphor im Sediment in erster Linie als Eisenphosphat vorhanden sein und wurde als solcher bestimmt, wobei die Probe zunächst mindestens 24 Stunden lang mit $\mathrm{KOH} \mathrm{(5 \% )}$ behandelt und dann geschüttelt und filtriert wird. Das Filtrat ist dann in bekannter Weise mit Ammonmolybdatlösung zu versetzen und die sich bildende tiefblaue Farbe wird anschließend im Zeiss-Elektrophotometer (Elko) in der 5-cm-Küvette erfaßt. Die Angabe der Ergebnisse erfolgt in $\mathrm{mg} / \mathrm{kg}$ Trockenmasse; die Fehlerbreite beträgt $\pm 1 \%$.

Bei der Betradhtung der in Abbildung 1 dargestellten Phosphat-Phosphor-Verteilung an der Sedimentoberfläche (etwa obere $10 \mathrm{~cm}$ ) des fraglichen Seegebietes ergibt 
sich sofort die evidente Beeinflussung durch die übergelagerten Wasserkörper aus der Elbe und vor allem aus der Alten Weser: Zwischen beiden Einflußzonen, etwa zwischen der Nordergründe-Tonne und der Weser-Schlüsseltonne, wurde mittelfeiner Sand von harter Konsistenz festgestellt, der mit der Ebbströmung aus dem Kerngebiet der Nordergründe-Sandbarren seewärts gelangt und der selbstverständlich relativ wenig Phosphat aufweist ( $\pm 20 \mathrm{mg} / \mathrm{kg}$ Trockensediment). Nach Westen, im Raume der eigentlichen Weserwasserzunge, d. h. etwa entlang der Verbindungslinie zwischen der Schlüsseltonne und dem Feuerschiff P 12, steigen die Werte auf das Doppelte an; dasselbe gilt für die Sedimente in nordöstlicher Richtung auf Elbe 1 und die Tonne $\mathrm{H} 1$ hin (Abb. 1). Dort kommen im Zentrum der Elbwasserzunge sogar Werte von über $50 \mathrm{mg} / \mathrm{kg}$ vor. Das sind recht hohe Beträge, wenn man sich vergegenwärtigt, daß z. B. die organisch stark belasteten Sedimente im Bremerhavener Fischereihafen einen Phosphatgehalt von "nur" 200-300 mg/kg aufweisen. Der relativ hohe Phosphatgehalt im Vorästuarsediment der beiden Ströme wird ohne weiteres verständlich durch die Tatsache des Aussedimentierens aus den mit Trübungspartikeln belasteten Astuarwasserkörpern und, im Falle der hohen Maxima vor der Elbmündung, durch das dortige, frïher bereits erwähnte Verklappen von biologisch geklärtem Abwasserschlamm der Großstadt Hamburg zwischen Elbe 1 und der Tonne H 1. - Diese Phosphatanreicherungen liegen um rund drei Zehnerpotenzen höher, als in den dazugehörigen Wasserkörpern, deren Phosphatgehalt sich auf etwa $30-50 \mu \mathrm{g} / 1$ beläuft. Sie stellen also eine beträchtliche Phosphatkonzentration aus dem Wasser dar, oder mit anderen Worten, das Sediment wird hier zu einem besonderen Phosphatdepot, das allerdings insofern nicht effektiv wird, als das $\mathrm{FePO}_{4}$ praktisch wasserunlöslich ist und höchstens dort wieder als freies $\mathrm{PO}_{4}$-Ion in Erscheinung tritt, wo es im Inneren der Sedimente $\mathrm{zu}$ vorübergehenden $\mathrm{pH}-\mathrm{Abnahmen}$ bis in schwachsaures Milieu kommt. Wir stellten aber in solchen Fällen immer wieder, auch noch in der reduktiven FeS-Zone in einigen $\mathrm{dm}$ Tiefen unter der Sedimentoberfläche beträchtliche Phosphatanreicherungen fest, die oft noch die Hälfte oder ein Drittel der Oberflächenkonzentration ausmachen. Das heißt aber, daß das Phosphat, wenn es erst einmal als $\mathrm{FePO}_{1}$ festgelegt ist, dem natürlichen Kreislauf zum Teil entzogen wird.

Abbildung 2 zeigt ein detailliertes Korngrößenbild aus dem Vorästuar der Elbe. Es entspricht in etwa dem in der vorhergehenden Darstellung gebrachten Eindruck der Elbebeeinflussung im Seegebiet westlich Elbe 1 mit Mehlsandcharakter von weit unter $100 \mu$ (bis ins Quasikolloide im Zentrum der Elbezunge) und mit gröberen Sedimenten (Dünensandcharakter von über $150 \mu$ ) südlich der 20-m-Linie in Richtung auf die Nordergründe und Alte Weser. Angesichts der Feinkörnigkeit sowie Belastung mit organischer Substanz ist der "Elbeklei“" in höchstens $1 \mathrm{~cm}$ Mächtigkeit durchoxydiert, während die Durchlüftung des Nordergründesandes $5 \mathrm{~cm}$ und mehr beträgt. Von Interesse ist noch die Tatsache, daß in dem tiefen Gebiet unmittelbar nördlich der 20-m-Linie die größeren Sedimentfraktionen über $300 \mu$ im Mikroskop als stark gerundete Quarzkörner erscheinen, die somit besonders „rollbar" sind, $d$. h. leidhter in der bodennahen Strömung vorankommen. Die Rundheit dieser Körner zeugt davon, daß sie sich bereits länger im oberflächennahen Geschiebe des Sedimentes aufhalten, und da sie südlich der 20-m-Linie nicht mehr beobachtet werden, ist anzunehmen, daß sie nicht etwa aus der Alten Weser herausgespiilt wurden, sondern sich wahrscheinlich seit langem 


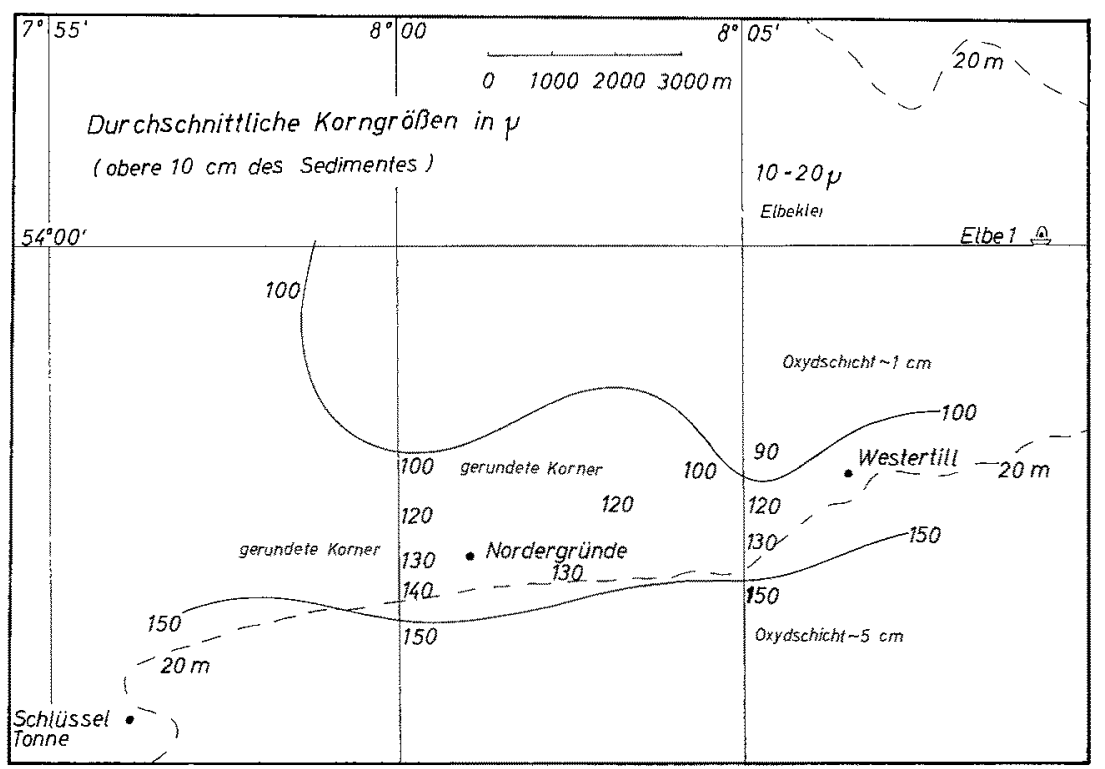

Abb. 2: Durchschnittliche Korngröße an der Sedimentoberfläche im Vorästuar von Elbe und Weser

im Einflußbereich des westlichen Reststromes am Nordsaum der holländischen und friesischen Küste aufgehalten haben und dort z. T. im Brandungsbereich der vorgelagerten Außensände nach und nach ihre typische abgerundete Form erhielten.

Aus der Alten Weser können diese Körner schon deshalb nicht stammen, weil im Zentrum der dortigen Fahrwasserrinne, auf Tiefen von über $20 \mathrm{~m}$, nachweislich das Pleistozän (Diluvium) angeschnitten wird; wir fanden nämlich beim Arbeiten mit dem van-Veen-Greifer häufig eisenüberzogene größere Geröllteile, Flintsteine, braunen Muschelschill usw. sowie grobkörnige Sande bis $1000 \mu$, die alle auf den Ursprung aus Pleistozänhorizonten hinweisen. Nach zahlreichen Binokularuntersuchungen (40fache Vergrößerung) sind diese Sedimente $\mathrm{n}$ i $\mathrm{cht}$ aus abgerundeten Körnern zusammengesetzt. Die Alte Weser, deren Pleistozäneinfluß im oberflächennahen Sediment nordwestlich der Nordergründe noch nachgewiesen wird, kann also nicht für die Existenz der erwähnten Rundkörner verantwortlich sein.

Ehe wir uns dem Einfluß aus der Alten Weser im einzelnen zuwenden sei in Abbildung 3 noch einmal anhand der Verteilung der gesamten organischen Substanz, wie sie durch nasse Verbrennung mittels $\mathrm{K}_{2} \mathrm{Cr}_{2} \mathrm{O}_{7}$ in der oxydativen Oberfläche des Sedimentes erfaßt wird, die Beeinflussung des Meeresbodens nördlich der Nordergründe aus der Elbmündung crläutert.

Wie nicht anders zu erwarten, koinzidiert das entsprechende Isolinienbild etwa mit dem der Körnungsisolinien und vor allem mit dem der Phosphatisolinien. Während der Sand der Nordergründe nur mit Spuren organischer Substanz belastet ist, nimmt der Gehalt derselben in nordöstlicher Richtung, nach Elbe 1 hin, schnell zu. In den Zonen kolloiden Schlickes nordwestlich von Elbe 1 stellten wir gelegentlich sogar 


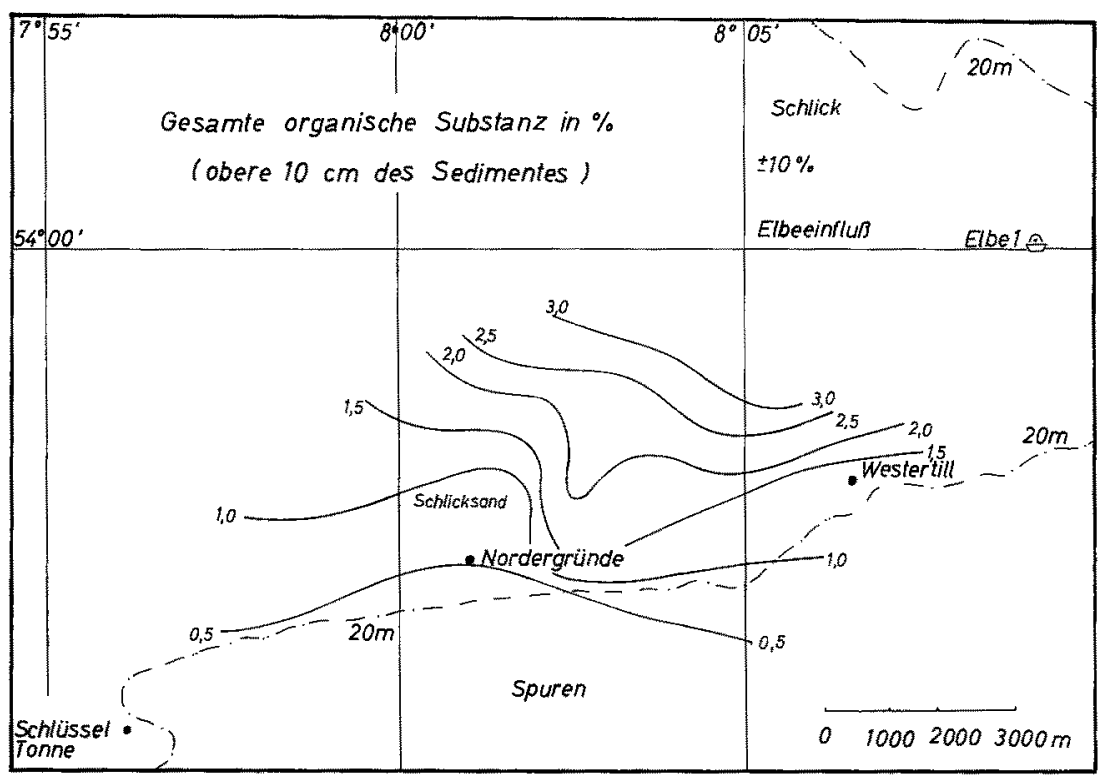

Abb. 3: Gesamte organische Substanz an der Sedimentoberfläche im Vorästuar von Elbe und Weser

Konzentrationen um $\pm 10 \%$ fest. Das ist eine bemerkenswerte Anreicherung verglichen mit dem durchschnittlichen Gehalt an der Oberfläche der gut eutrophierten Watten des inneren Weserästuars von nur $\pm 2 \%$.

In Abbildung 4 kommt noch (wieder nordöstlich der Nordergründetonne) im Gehalt des sog. „mobilen“ Eisens - das ist das in oxydischer oder auch sulfidischer Form in vorwiegend kolloidem Zustand vorliegende und in geringerem Ausmaße auch im $\mathrm{FePO}_{4}$, nicht aber in den Mineralen der Sandlkörner, gebundene, also relativ bewegliche und als $\mathrm{Fe}^{+++}$über Ammonzitrat erfaßte Eisen - der ausgeprägte Zusammenhang zwischen feinstkörnigen, tonigen Sedimenten und der Konzentration des erwähnten Eisens zum Ausdruck. In Richtung auf den „Elbeklei“ nimmt der Eisengehalt schnell zu und erreicht nordwestlich Elbe 1 Spitzenwerte von etwa 20\%, d. h. etwa das Vierfache der entsprechenden Eisenwerte an der Oberfläche der inneren Weserwatten und analoger Sedimente. Auf den Nordergründen selbst beläuft sich der Eisengehalt dagegen nur auf $\leqq 1 \%$. Dieser Verteilung kann man also entnehmen, daß der Gehalt an "mobilem" Eisen generell der Korngröße des Sedimentes umgekehrt proportional ist.

Im entgegengesetzten Sinne verläuft der Gradient des zweiten auf derselben $A b-$ bildung 4 zur Darstellung gebrachten Eisenfaktors, nämlich der Anzahl der sog. Braunkörner (rostbrauner, eisenoxydig inkrustierter Sandkörner pro $\mathrm{cm}^{2}$ der Sedimentoberfläche). Diese Braunkornzahl nimmt, von der Nordergründetonne aus betrachtet, nach Südwesten am Westende der Gründe und damit in Richtung auf die Alte Weser kontinuierlich $\mathrm{zu}$.

Bevor aber dieser besondere Isolinienverlauf eindeutig als Einfluß aus der Alten- 
Weser-Rinne ausgelegt werden kann, ist es erforderlich, die natürlichen Voraussetzungen für diese "rostigen" Sandkörner sowie die Methode ihrer quantitativen Bestimmung und ihre Bedeutung für die Erfassung der oberflächennahen Bodenbewegung näher zu erläutern. Bekanntlich läßt sich die Bewegung der Sedimentoberfläche auf

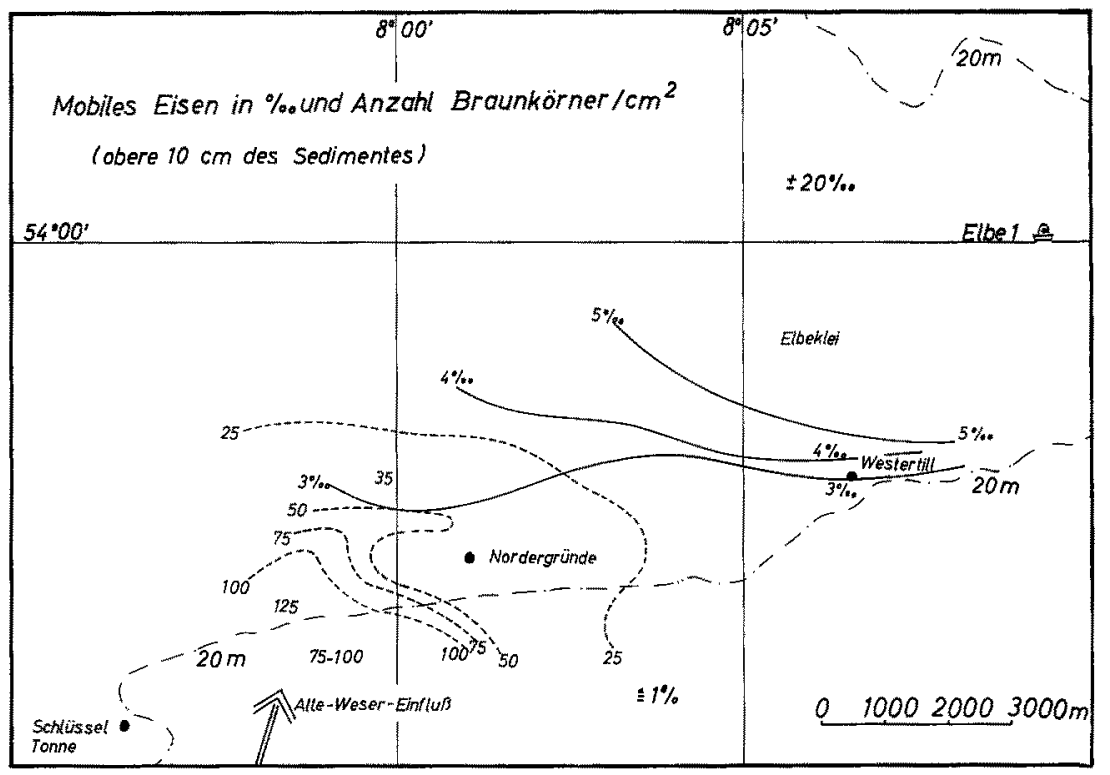

Abb. 4: Gehalt an mobilem Eisen in \% sowie Anzahl der sogen. Braunkörner $/ \mathrm{cm}^{2}$ an der Sedimentoberfläche im Vorästuar von Elbe und Weser

grundsätzlich sehr verschiedenen Wegen erfassen, und zwar zunächst einmal am effektivsten mit Hilfe radioaktiver Isotopen, der sog. "tracer elements“, ein Verfahren, daß aber sowohl in finanzieller als auch in personeller Hinsicht recht aufwendig ist. Oder man arbeitet mit relativ wasserresistent eingefärbtem Originalsand der Untersuchungsstation (z.B. mit Methylenblau oder auch mit Luminophoren gefärbt), ein Verfahren, das eigentlich nur dort wirkungsvoll eingesetzt werden kann, wo der Meeresboden, wie auf den Watten, in regelmäßigen Zeitabständen trockenfällt und somit das gefärbte Material leicht auszubringen und wieder einzusammeln ist. Schließlich kann man sich aber auch noch mit Erfolg natürlicher Leitfaktoren aus dem Sediment selbst bedienen, wie sie sich bei uns im litoralen Vorfeld auch für den sedimentpedrographisch nicht geschulten Fachmann gerade in der Gestalt von eiseninkrustierten Sandkörnern anbieten, die in den tiefen Rinnen aus submersen, erodierten Pleistozänhorizonten freigespült und, der Bodendynamik entsprechend, verteilt werden.

Die in diesem Zusammenhang bedeutungsvolle Pleistozänoberfläche liegt im äußeren Teil des Weserästuars in ca. $20 \mathrm{~m}$ Tiefe unter S.K.N. und wird ganz besonders in der Alten Weser nördlich des Roten-Sand-Leuchtturmes in einer etwa 6-7 km langen Erosionsrinne angeschnitten. Pleistozänschichten weisen nun häufig wenige Meter unter ihrer Oberfläche oxydative Eisenverdichtungen, den sog. „Ortstein “ auf, das sind An- 
reicherungen von eisenooly thisch verk rusteten - umhüllten-Sandkörnern und sonstigen Eisenkonkretionen (gelegentlich dunkle Manganverbindungen einschließend). Sie werden, wie schon erwähnt, zusammen mit grobem Geröll, Flintsteinen usw. in den Außenrinnen freigespült. - Bei den zur Zeit (Ende 1963) noch laufenden Arbeiten in den tiefen Außenrinnen stellten wir übrigens auch noch fest, daß in diesen pleistozänen Erosionsrinnen vorhandener Muschelbruch (Schill) durch noch zu klärende Vorgänge durch und durch braun, d. h. eisenhaltig ist, und zwar nicht nur an der Oberfläche der einzelnen Bruchteile, sondern auch im inneren Gefüge derselben. Es mag sich hierbei um eine rein mechanische Adsorbtion koloiden Eisens handeln.

Der Eisengehalt solcher Muschelschalen liegt nach eigenen Feststellungen bei Werten bis zu 5\% im Trockensediment, das ist eine Größenordnung, wie sie auch im festländischen Ortstein von uns angetroffen wurde. - Die gewöhnlich in reduktiven Wattenhorizonten lebenden Muscheln und ihre freigespïlten Schalen sind höchstens stellenweise an einzelnen meist kleineren Flächen schwarz oder braun gefärbt und werden auch später im durchlüfteten Seewasser nie völlig braun; die Vorgeschichte der Muschein im Watt kann also auf keinen Fall verantwortlich gemacht werden für das Phänomen des durch und durch braunen Schills, wie er unter anderem hier am Roten Sand angetroffen wird. Nach eigenen Befunden und weiteren Erhebungen kommt ein solcher Braunschill, dessen Kleinstpartikel ebenfalls bei der Bestimmung der Braunkornzahl berïcksichtigt werden, nur dort in der südlichen Nordsee vor, wo submerse Pleistozän- oder Tertiärhorizonte unter Erosion stehen, also bevorzugt in der Alten Weser, dann aber unter Umständen noch in der Robinsbalje und der tiefen Tillrinne in den äußeren Wurster Watten.

Zur quantitativen Bestimmung der Braunkornzahl $/ \mathrm{cm}^{2}$ Sedimentoberfläche wurde das nachfolgende Verfahren von uns eingeführt: Die mehr oder minder eisenoolytisch (rostig) eingeschlossenen Sandkörner und opak-rostigen Sedimenttrümmer der gängigen Durchschnittskorngröße zwischen 100 und $300 \mu-$ Körner unter $100 \mu$ sind für die Beobachtung zu klein und bei Körnungen über $300 \mu$ ist die Kornanzahl insgesamt zu gering - werden nach ammioniakalischer Auswaschung (Beseitigung des Salzgehaltes) und nach Behandlung mit Isopropylalkohol (Beseitigung des Aneinanderklebens der mit organischer Substanz behafteten Körner) in trockenem Zustand auf einer Zählplatte mit Quadratzentimetereinteilung bei hellster Beleuchtung im Binokular (40fache Vergrößerung) ausgezählt und die einzelnen Auszählungen dann nach der Anzahl vorhandener $\mathrm{cm}^{2}$-Felder gemittelt. - Um zu erreichen, daß der durch die Vorbehandlung gelockerte Sand in einer Schichtdicke von nur einem Korndurchmesser auf der Platte lückenlos nebeneinanderliegt, wird diese dünn mit Glyzerin bestrichen, der Sand dann gleichmäßig darüber hingestreut und so weit abgeblasen, daß er nur in „einkörniger" Schicht darauf liegen bleibt. Die rostigen Körner werden auch dann noch mitgezählt, wenn sie nur zu einem gewissen Bruchteil eisenoolythisch umhüllt sind, und zwar bis zu etwa 10\% der Umrißfläche des betreffenden Sandkornes; denn ein großer Teil der rostigen Körner, die zunächst gleich nach ihrer Erosion

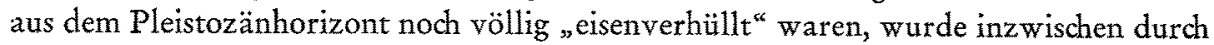
das bekannte Rollen und Springen in der bodennahen Strömung mehr oder minder von der Eisenhülle befreit, muß aber dennoch als eisenoolythisch bezeichnet und mitgezählt werden. 
Die so ermittelte „Braunkornzahl“ (Streuung \pm 5 Einheiten) stellt nach unseren bisherigen Erfahrungen im Gebiet der Alten Weser und seewärts davon einen repräsentativen und schnell erfaßbaren Ausdruck für den Einfluß der erwähnten Pleistozänhorizonte auf das benachbarte und seewärtige Sediment dar. - Es sei an dieser Stelle darauf hingewiesen, daß dieses Referat lediglich den momentanen Stand (Oktober 1963) der entsprechenden Untersuchungen bringt und daß somit die diskutierten Darstellungen erste Zusammenhänge aufzeigen und in Zukunft noch durch analoge Untersuchungen vor anderen Erosionsrinnen im Elbe-Weser-Winkel ergänzt werden sollen.

Nach diesen grundsätzlichen Darlegungen des Prinzips, der Methodik und Bedeutung des Braunkorn-Verfahrens kehren wir zurück zum Ausgangspunkt der Betrachtungen, nämlich der Verteilung der Braunkornzahl im Vorfeld der Alten Weser, die als der Hauptabflußarm des gesamten Weserästuars zu gelten hat und aus dynamischen Gegebenheiten dieses Vorfeld sowohl hydrographisch als auch sedimentologisch ausfächernd beeinflußt.

Es ist bekannt, daß ein östlicher Zweig der Alten Weser häufig zwischen der Schlüsseltonne und der Nordergründetonne im Uhrzeigersinn nach Nordost abdreht und mit einem entsprechenden Materialtransport verbunden ist. Nach den bisherigen Erfahrungen mit der Braunkornmethode in der Zeit zwischen Frühjahr und Herbst

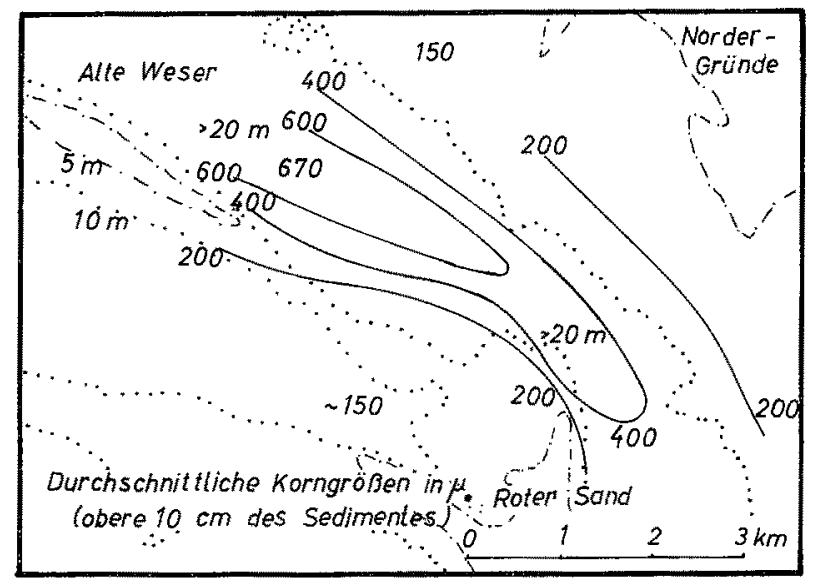

Abb. 5: Durchschnittliche Korngröße an der Sedimentoberfläche im äußeren Weserästuar

1963 sowohl in Landnähe als auch in Landferne des Weserästuars sind Braunkornzahlen uber 50 und bis zu 100 und mehr Einheiten als relativ hoch zu betrachten, während an erosionsferneren Stationen Werte unter 50 und bis zu 10 Einheiten oder gar bis zu Spuren herab als Ausdruck geringer Konzentration zu werten sind.

Nach Voraussendung dieser Tatsachen ergibt sich im nördlichsten Vorfeld der Alten Weser, im Gebiet der Nordergründetonne ( $\mathrm{Abb} .4$ ) eine nur geringe Braunkornanreicherung, die aber von dort in Richtung Südwest, nach der Schlïsseltonne hin stets um das $Z_{w e i-}$ bis Dreifache, d. h. auf 100 Einheiten und mehr, ansteigt, eine Erscheinung, die im allgemeinen weiter nach Westen hin wieder ablklingt, so daß man hier unbedingt von einer Sedimentbeeinflussung aus dem Süden, d. h. aber aus der Rinne 
der Alten Weser sprechen kann. Dieses Phänomen wird bei der Betrachtung der Braunkornverteilungen in der Alten Weser selbst recht gut evident.

Die entscheidenden Sedimenteigenschaften des äußeren Vorfeldes der Alten Weser (Nordwestrand der Nordergründe) findet man in etwa gleicher Konzentration in der 8-10 km südöstlich, in Stromluv belegenen Erosionsrinne der Alten Weser und

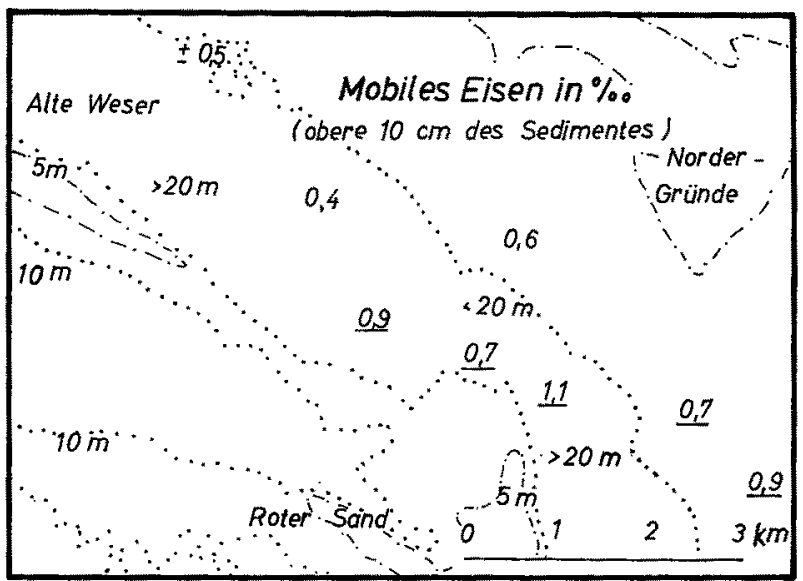

Abb. 6: Gehalt an mobilem Eisen in \%o an der Sedimentoberfläche im äußeren Weserästuar

ihrer unmittelbaren Umgebung wieder, wie es zunächst einmal aus der Verteilung der durchschnittlichen Korngröße in Abbildung 5 hervorgehen mag. - Man beobachtet dort auf beiden Seiten der Rinne, d. h. am Roten Sand und auf den südlichen Nordergründen, Korngrößen um 150-200 $\mu$, wie sie schon am Nordwestzipfel der Nordergründe angetroffen wurden. Innerhalb der $10-\mathrm{m}$-Linien, also zum Zentrum der Rinne hin, nimmt die Korngröße gleichmäßig auf 200-400 $\mu \mathrm{zu}$, um im tiefsten Teil derselben, in der eigentlichen Erosionszone, bei Tiefen über $20 \mathrm{~m}$, Durchschnittskörnungen von weit über $600 \mu$, in Einzelfällen bis $1000 \mu$ und mehr zu erreichen. Derart große Körnungen zeugen von der Pleistozänerosion in der am Nordende des Roten Sandes entlang der südlichen 10-m-Linie beginnenden und bis zur 5-m-Bank am Südwestrand der Alten Weser reichenden Rinne. Diese Größtkörner werden nach jüngsten Beobachtungen nicht in das Gebiet zwischen der Alten Weser und deren nordwestlichem Vorfeld oder gar in dieses selbst transportiert.

Wie vor der Elbmündung interessiert auch hier in der Alten Weser im Anschluß an die Korngröße der Gehalt an adsorbiertem (mobilem oder kolloidem) Eisen im Oberflächensediment, wie er in Abbildung 6 zur Darstellung kommt. - Die Unterschiede im Eisengehalt des Sedimentes sind hier im reinen Sandbereich relativ gering, so daß eine repräsentative Isoliniendarstellung kaum in Frage kam. Das hat seine Ursache darin, daß der Eisengehalt in den sandigen Außengebieten infolge der Grobkörnigkeit und damit fehlenden Adsorbtionskraft absolut betrachtet sehr gering ist (meist kleiner als $1 \%$ ). Dennoch kann man der in Abbildung 6 angegebenen Verteilung entnehmen, daß der Eisengehalt im Zentrum der Erosionsrinne nördlich und nordöstlich des Roten Sandes mit $\pm 1 \%$ Fe i. Tr. im Verhältnis zu den braunkorn- 
armen Sänden der Seitengebiete etwa doppelt so hoch liegt wie auf denselben, und das kann nur seine Ursache in der relativen Eisenanreicherung der Pleistozänrinne haben, worauf die dort stets angetroffenen "rostigen " Flintsteine hindeuten, aber auch ganz besonders noch die Verteilung der Braunkornzahl, wie sie in der Abbildung 7 dargestellt ist. Im Nordosten, beiderseits des Roten Sandes, treten im Ostende der Erosionsrinne Braunkornzahlen um 60-70 Einheiten und auch noch darüber auf, während sie in den Seitenräumen auf etwa die Hälfte herabgehen. Der Zusammenhang zwischen der Braunkornzahl und der Pleistozänerosion ist hier ohne weiteres ersichtlich.

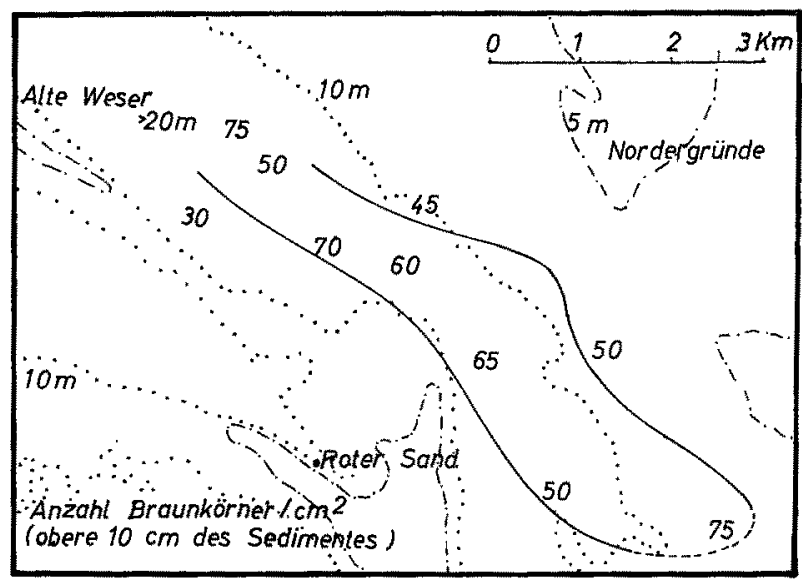

Abb.7: Anzahl der sogen. Braunkörner/ $/ \mathrm{cm}^{2}$ an der Sedimentoberfläche im äußeren Weserästuar

In jüngster Zeit getätigte Erkenntnisse erhärten die bisher diskutierten Befunde noch insofern, als sich die entsprechenden Analysenergebnisse aus dem 5-8 $\mathrm{km}$ langen Seebereich zwischen dem Nordwestende der Nordergründe (Abb. 1-4) und dem Zentrum der Alten Weser folgerichtig an ihre beiden Nachbarräume anschließen:

Das geht aus der Abbildung 8 hervor, in der die Verteilung der Braunkornzahl im Bereich der Erosionsrinne selbst, aber auch in Westteil der Alten Weser bis zum Außenende der Nordergründe dargestellt ist. - Am Verlauf der 50 Braunkorn- und der 100 Braunkornisolinie wird der unmittelbare Zusammenhang zwischen dem Zentralgebiet der Alten Weserrinne und ihrem seewärtigen Vorfeld ohne weiteres klar. Die aus der Rinue herausgespülten eisenhaltigen Sedimenttrümmer reichern sich zunächst im Westteil der Alten Weser an (in der eigentlichen Rinne Werte um 60-70 Einheiten, westlich davon, bis ins Gebiet der Schlüsseltonne, 100 Einheiten und mehr). Das Zentrum der Braunkornkonzentration biegt dann östlich der Schlüsseltonne nach Norden ab und fügt sich somit den am Anfang der Sommersaison 1963 gewonnenen Isolinienbildern gut ein.

Übrigens entsprechen auch die durchschnittlichen Korngrößen sowie die Konzentrationen an mobilem Eisen in dem erwähnten $Z$ wischengebiet völlig den zugehörigen Braunkornwerten: Denn in der eigentlichen Erosionsrinne liegen die durchschnittlichen Korngrößen noch bei $300 \mu$ und darüber, um dann in Richtung West und Nordwest auf etwa $150 \mu$, dem charakteristischen Wert der westlichen Nordergründe, ab- 


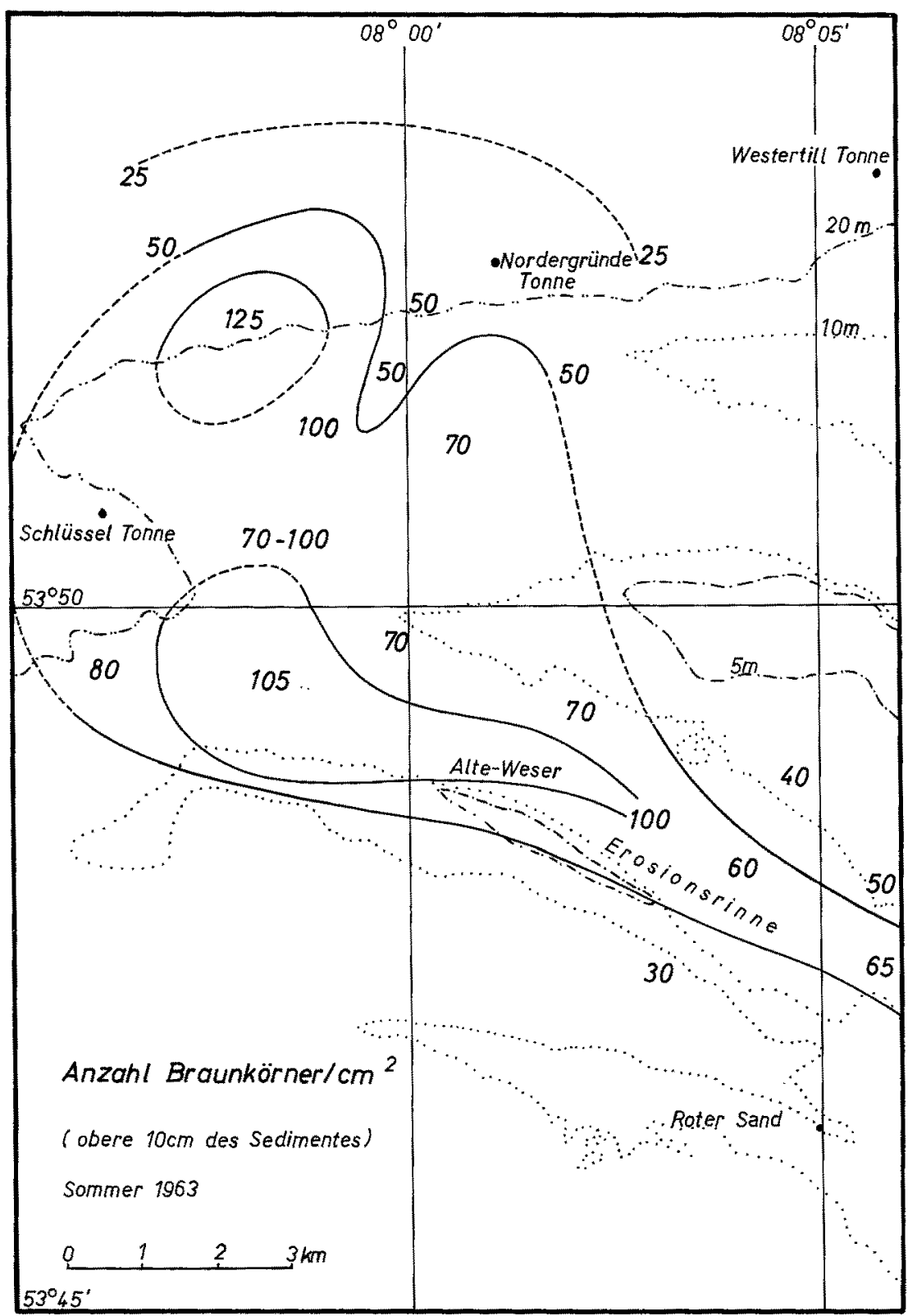

Abb. 8: Anzahl der sogen. Braunkörner $/ \mathrm{cm}^{2}$ an der Sedimentoberfläche im äußeren Weserästuar und im Seegebiet nordwestlich desselben

zusinken. Und der Gehalt an mobilem Eisen liegt im maximalen Braunzahlgebiet südöstlich und östlich der Schlüsseltonne, bei etwa 1,5\% Fe i. Tr., das ist aber ein für die „oligotrophen“, also chemisch kaum belasteten Außensände relativ hoher Wert. In 
derart reinen Außensänden ist der Gehalt an mobilem Eisen also weitgehend abhängig von der Gegenwart der Braunkörner, d. h. aber von der Nähe eines PleistozänErosionshorizontes. Das Eisen wird daher in dieser Form ein einfaches und zuverlässiges "tracer-element" für die Bewegungsrichtung der Sedimentoberfläche in der Nähe von Pleistozänerosionen und deutet darüber hinaus auf diese hin. - Nach unseren Vermutungen wird ebenfalls in der über $20 \mathrm{~m}$ tiefen Erosionsrinne der westlichen Till (südlich des Scharhörnrifts), in etwa gleichem Küstenabstand wie bei der Alten Weser, das Pleistozän angeschnitten. Um mehr Aufschluß in diesem Zweig des Eisenkreislaufes im Bereich der Vorwatten zu erhalten, soll in Kürze auch dieser Vorästuarraum systematisch aufgesucht und auf seinen speziellen Eisenhaushalt untersucht werden.

Diese vorläufigen Ausführungen abschließend sei noch einmal festgestellt, daß man in der Braunkornzahl bei gegebener Voraussetzung ein weiteres und dabei wenig aufwendiges Mittel anhand hat, die Wanderung des Sedimentes und seine Beeinflussung aus dem Litoral zu ermitteln. Und schließlich scheint es besonders bemerkenswert, daß aus den erwähnten Pleistozän-Erosionsstellen heraus (wie weit diese evtl. auch weiter draußen in der offenen See anzutreffen sein werden, entzieht sich noch meiner Kenntnis) sekundär noch einmal Eisen in das Milieu der Vorästuare gelangt, nach dem dieses fast restlos in den Sedimenten der inneren Watten durch Flockung und Adsorption festgehalten wurde und sonst höchstens noch in hochviskosen und kolloidfeinen Schlickablagerungen in tieferen Mulden der offenen See (wenn auch langsamer sedimentiert) stärker konzentriert vorkommt. In bezug auf den Eisenhaushalt - in diesem Zusammenhang sei auch auf die Bedeutung des kolloiden Eisens als $A$ bbinder für $\mathrm{PO}_{4}$ in Form von $\mathrm{FePO}_{4}$ hingewiesen - stellen die hier behandelten Pleistozän-Erosionsrinnen einen Fremdkörper in dem Bereich der oft völlig eisenfreien Außensände und Vorästuare dar.

\section{ZUSAMMENFASSUNG}

1. Im Anschluß an frühere systematische Untersuchungen über die Wassermischvorgänge vor den Weser- und Elbästuaren wurden im Sommerhalbjahr 1963 im gleichen Raume sedimentologische Arbeiten durchgeführt, die zur Lösung von Fragen der offensichtlichen Beeinflussung der oberllächennahen Sedimente aus den beiden markanten Astuarwasserkörpern beitragen können. Hierbei wurde gleichzeitig im Bereich des Eisenchemismus der Bodenoberfläche eine Beeinflussung aus Pleistozänhorizonten in den über $20 \mathrm{~m}$ tiefen Außenrinnen der Vorästuare evident.

2. Die Verteilung des anorganischen Phosphats ( $\mathrm{PO}_{4}-\mathrm{P}$ in $\mu \mathrm{g} / \mathrm{kg}$ Trockensediment) im Sediment nordwestlich des Feuerschiffes Elbe 1 und südöstlich des Feuerschiffes P 12 läßt in beiden Gebieten jeweils den Einfluß aus dem Elbe- und aus dem Weserästuar offenbar werden. Nach einem besonderen Verfahren wird hierbei das adsorbierte und vor allem das am Eisen in $\mathrm{FePO}_{4} z$. T. irreversibel gebundene $\mathrm{PO}_{4}$ erfaßt. Der Phosphatgehalt beträgt im Zentrum der beiden Astuarsedimentzungen (bei Elbe 1 und bei P 12) etwa $50 \mathrm{mg} \mathrm{PO}_{4}-\mathrm{P} / \mathrm{kg}$, während die entsprechenden Werte in den nicht aus den Astuaren beeinflußten Zwischengebieten auf etwa die 
Hälfte herabsinken. Der Gehalt an $\mathrm{PO}_{4}-\mathrm{P}$ im Sediment liegt übrigens um etwa drei Zehnerpotenzen höher als in den überlagerten Wasserschichten. Es wird noch auf die besondere "Eutrophierung" des Sedimentes westlich von Elbe 1 eingegangen, die thre Ursache vor allem in der dortigen Verklappung von Abwasserschlamm der Großstadt Hamburg hat.

3. Die Korngrößenverteilung im Seegebiet vor den Nordergründen, also in den Vorästuaren von Elbe und Weser, spiegelt ebenfalls in etwa die Beeinflussung der oberflächennahen Sedimentschichten aus den übergelagerten beiden Wasserzungen wieder, aus denen feinkörnige Sinkstoffe von ca. $\pm 50 \mu \mathrm{m}$ Teilchendurchmesser $\mathrm{zu}$ Boden rieseln und zwischen denen (auf den nordwestlichen Nordergründen selbst und im Bereiche der Nordergründe-Tonne) die Sedimentkörnung zwei- bis viermal größer ist. Die durchschnittliche Körnung in den Ästuarsedimentzungen beträgt ca. $\leqq 50 \mu \mathrm{m}$, in den Zwischenzonen etwa 100-200 $\mu \mathrm{m}$. Die Sandkörner in diesen Zwischenzonen weisen oft einen hohen Grad an Rundheit (Rundschliff) auf, d. h. sie haben eine längere marine Vorgeschichte als die Körner in den Astuarrinnen selbst.

4. Es folgt die Verteilung der gesamten organischen Substanz in den oberflächennahen, durchoxydierten (etwa $1-5 \mathrm{~cm}$ mächtige Oxydation) Schichten, wie sie auf dem Wege der „nassen Verbrennung" mit $\mathrm{K}_{2} \mathrm{Cr}_{2} \mathrm{O}_{7}$ ermittelt wurde. Im Zentrum der Astuarsedimentzungen und hier besonders im Kerngebiet der Elbezunge nordwestlich Elbe 1 steigen die Werte auf etwa 10\% organische Substanz i. Tr. an, ein Maximum, das sich ohne weiteres aus dem Hamburger Abwasserschlamm erklärt. In den Zwischengebieten sinken die entsprechenden Werte dann oft auf Spuren herab.

5. Das sogenannte mobile Eisen, das ist das an die Sedimentpartikel adsorbierte oder zum geringen Teil auch im $\mathrm{FePO}_{4}$ gebundene Eisen (nicht etwa auch noch das in den Mineralen fest gebundene Eisen), das nach einem besonderen Verfahren mittels Ammonzitrat aus dem Sediment geholt wird, ist in den feinkörnigen Astuarsedimenten wesentlich mehr angereichert als etwa in den sandigen Zwischenpartien. So belaufen sich z. B. die entsprechenden Werte in den Ästuarzungen auf 10-20\% $\mathrm{Fe} / \mathrm{kg}$ i. Tr. und in den Zwischenzonen nur auf ca. 1\% und weniger. Die Feinstsedimente adsorbieren wesentlich größere Mengen dieses Eisens. Die sandigeren Sedimente auf den Nordergründen werden in ihrem geringen Eisengehalt weitgehend gesteuert von absolut betrachtet nur geringen pleistozänen Eisenvorkommen, die aber repräsentativ sind für eine ganz typische Verteilung eisenoolithisch umhüllter Sandkörner, welche in ihrer Existenz nicht nur hinweisen auf erodierte Pleistozänhorizonte, sondern darüber hinaus auch noch als Indikator für die Sedimentwanderung in der Nähe (10-20 km Umkreis) solcher Erosionsrinnen gelten. Die quantitative Erfassung dieser „rostigen “ Indikator-Sandkörner erfolgt durch Auszählung auf einer $\mathrm{cm}^{2}$-Zählplatte im Binokular bei 40 facher Vergrößerung nach besonderer Vorbehandlung des betreffenden Sandes. Im Verlaufe der bisherigen Untersuchungen wurde bereits festgestellt, daß die sogenannten „Braunkornzahlen $/ \mathrm{cm}^{2}$ Sandoberfläche" im Bereich von 0 bis etwa 40 Einheiten darauf hinweisen, daß der ortsteinhaltige Erosionshorizont etwa $20 \mathrm{~km}$ und mehr vom Beobachtungspunkt entfernt ist. Bei Werten von über 50 und bis über 100 Einheiten befindet man sich in der Nähe solcher Eisenoolithe liefernden Horizonte. 
6. In mehreren Darstellungen folgt abschließend die Verteilung der Sedimentkörnungen, des Gehaltes an mobilem Eisen und der aufschlußreichen Braunkornzahlen aus dem Gebiet der tiefen Alten Weser-Rinne nördlich des Roten-Sand-Leuchtturmes sowie ihres Vorfeldes am Westende der Nordergründe. Aus den Isolinienbildern geht eindeutig hervor, wie nicht nur die Korngröße selbst, sondern auch der Gehalt an mobilem Eisen aus der Pleistozänrinne heraus beeinflußt wird. Die Körnung steigt im Bereich der Rinne auf 600 und u. U. gar $1000 \mu \mathrm{m}$ und mehr an, wobei gleichzeitig im Bodengreifer häufig "rostige" Flintsteine und anderes Pleistozängeröll zutage tritt. In der Erosionsrinne beläuft sich ferner der Gehalt an mobilem Eisen auf $\pm 1 \%$ Fe i. Tr., während die feinkörnigeren (aber nicht schlickigen) Sedimente in den Seitenräumen der Alten Weser nur noch Spuren an Eisen aufweisen. Und schließlich deutet der Verlauf der Braunkornisolinien mit über 100 Einheiten $/ \mathrm{cm}^{2}$ in und vor der Rinne und in einem weiten Ausholen dieser Isolinie nach Nordwesten bis an die 20-m-Linie vor den Nordergründen auf die Beeinflussung der Sedimentoberfläche aus der Erosionsrinne hin.

\section{Diskussion im Anschluß an den Vortrag LüNEBURG}

CAspers: Wir beobachten in der Außenelbe weiche, zum Teil recht flüssige Sedimentauflagen, die für die Benthos-Verteilung von Bedeutung sind. Haben Sie bei Thren Untersuchungen diese Auflagen mitberüdksichtigt?

LüNEBURG: Wir haben sie nicht berücksichtigt, sondern weggespült und nur das harte Sediment untersucht. Weiter in den Watten sind wir dann aber noch tiefer bis auf 30 oder $40 \mathrm{~cm}$ gegangen, in die reduktiven Horizonte hinein. Es wäre vielleicht noch nachzutragen, daß die Phosphatverteilung in der Vertikalen recht interessant ist. Sie nimmt nämlich sehr langsam ab; erstaunlicherweise findet man auch im FeS- und im FeS2-Horizont noch immer große Phosphatmengen, etwa die Hälfte oder ein Drittel der Oberflächenwerte. Ich hatte ursprünglich vermutet, daß das Phosphat wieder frei wird, aber es ist ja recht unlöslich und wird noch unlöslicher als Ferro-Phosphat; es geht kaum wieder in das Grundwasser der Sedimente zurïck. Wir haben das Grundwasser für sich durch Abschleudern untersucht. Es ist recht phosphatarm; vermutlich wird das Phosphat "für immer" niedergeschlagen.

GunkEL: Ist untersucht worden, ob Eisenbakterien eine Rolle bei der Ausfällung von Eisen bei den sogenannten Braunkörnern spielen?

LÜNEBURG: Nein. Das setzt ein team-work mit Bakteriologen voraus. Sicher wäre so etwas anzustreben.

CASPERS: Liegt Ihr Untersuchungsgebiet noch im Aestuarbereich? Es ist durch die Aestuare beeinflußt, stellt also ein "Vormündungsgebiet" dar, aber der Begriff "Aestuar" sollte auf das Flußgebiet beschränkt bleiben.

LüNEBURG: Das kann man biologisch oder topographisch definieren. Topographisch geht es bis Helgoland. Helgoland liegt dann am "rechten Elbufer", und hydrographisch wird das Gebiet vor Helgoland ja tatsächlich oft von der Elbe beeinflußt. Das Elbwasser reicht häufig bis Helgoland hinaus. Wenn man das Aestuarwasser hydrographisch definiert, könnte man sich vielleicht des Venedig-Systems, an dem Sie ja selbst mitgearbeitet haben, bedienen und dann sagen, daß jenseits von $30 \%$ das marine Brackwasser aufhört. 